\title{
Redesign of Plates by Large Admissible Perturbations
}

\author{
Michael M. Bernitsas* and Chae Whan Rim ${ }^{\dagger}$ \\ University of Michigan, Ann Arbor, Michigan 48109
}

\begin{abstract}
The problem of structural redesign of plates for static deflection and modal dynamics objectives is formulated and solved by the method of large admissible perturbations. The perturbation approach to redesign is first used to develop response equations for the objective plate design based on its specifications and the baseline plate design. The equations of the objective state are strongly nonlinear implicit expressions of the variable plate thickness. A large admissible perturbations algorithm is developed to solve the plate redesign problem and define the optimal objective state. The latter is reached incrementally with a prediction-correction scheme without repeated finite element analyses. Systematic numerical applications in redesign of a cantilever plate of 216 degrees of freedom are used to investigate the effects of number of extracted modes and redesign variables. It is shown that the large admissible perturbations theory can be used efficiently to redesign plates for multiple specifications that require changes to the baseline design and its response of the order of $100 \%$.
\end{abstract}

\section{Nomenclature}

$c_{i j} \quad=$ admixture coefficient for participation of the $j$ th mode to changes in the $i$ th mode

$E \quad=$ Young's modulus

$[k],[K \backslash]=$ global and generalized stiffness matrices

$\left[k_{e}\right]=$ stiffness matrix of element or group of elements related to property $e$

$K_{i} \quad=i$ th component of [ $K$ \]

$[\mathrm{m}],\left[M_{\backslash}\right]=$ global and generalized mass matrices

$\left[m_{e}\right] \quad=$ mass matrix of element or group of elements related to property $e$

$M_{i} \quad=i$ th component of [ $M$, ]

$n \quad=$ number of degrees of freedom in finite element model

$N=$ number of increments in incremental algorithm

$n_{a} \quad=$ number and admissibility constraints

$n_{u}, n_{\omega}, n_{\phi} \quad=$ number of displacement, frequency, and mode shape constraints

$n_{r} \quad=$ number of extracted structural modes used in program for redesign of structures

$p \quad=$ number of redesign variables

$S 1, S 2 \quad=$ initial and objective structural states, respectively

$\{u\} \quad=$ nodal static displacement vector

$\alpha_{e} \quad=$ fractional change to element or group property $e$

$\alpha_{e}^{+}, \alpha_{e}^{-} \quad=$ upper and lower bounds of $\alpha_{e}$

$\Delta \quad=$ prefix denoting total change

$\delta=$ prefix denoting incremental change

[ф] $\quad=$ matrix of mode shape vectors

$\phi_{k i} \quad=k$ th degree of freedom $i$ th mode shape

$\{\psi\}_{i} \quad=i$ th mode shape

$\omega_{i} \quad=i$ th natural frequency

Indices and Special Symbols

$e \quad=$ index for redesign variables

$\ell=$ denoting quantity in increment $1,2, \ldots, N$

[ ] = diagonal matrix

()$^{\prime} \quad=$ structural property of state $S 2$

\section{Introduction and Background}

S TRUCTURAL perturbation methods have been developed since $1975^{1,2}$ and have been used to solve several challenging structural analysis and design problems. Linear perturbation was introduced by Stetson ${ }^{1}$ and Stetson and Harrison, ${ }^{2}$ and modified by Sandström and Anderson ${ }^{3}$ for redesigning a structure to improve its modal dynamics. Both natural frequency and mode shape improvements were achieved. The meaning of linear here is that differences in redesign variables and response between the initial and the improved structural states are small. Nonlinear perturbation methods ${ }^{4-17}$ are used to find the objective state for large differences-of the order of $100 \%$-between the two structural states. Perturbation methods calculate the objective state without trial and error or repeated finite element analyses (FEAs) by postprocessing the FEA results of the initial design. Presently, research in nonlinear perturbation methods follows several paths which are summarized in Fig. 1 and outlined hereafter.

The primary goal of nonlinear perturbation methods is to formulate and solve structural analysis and design problems as two-state

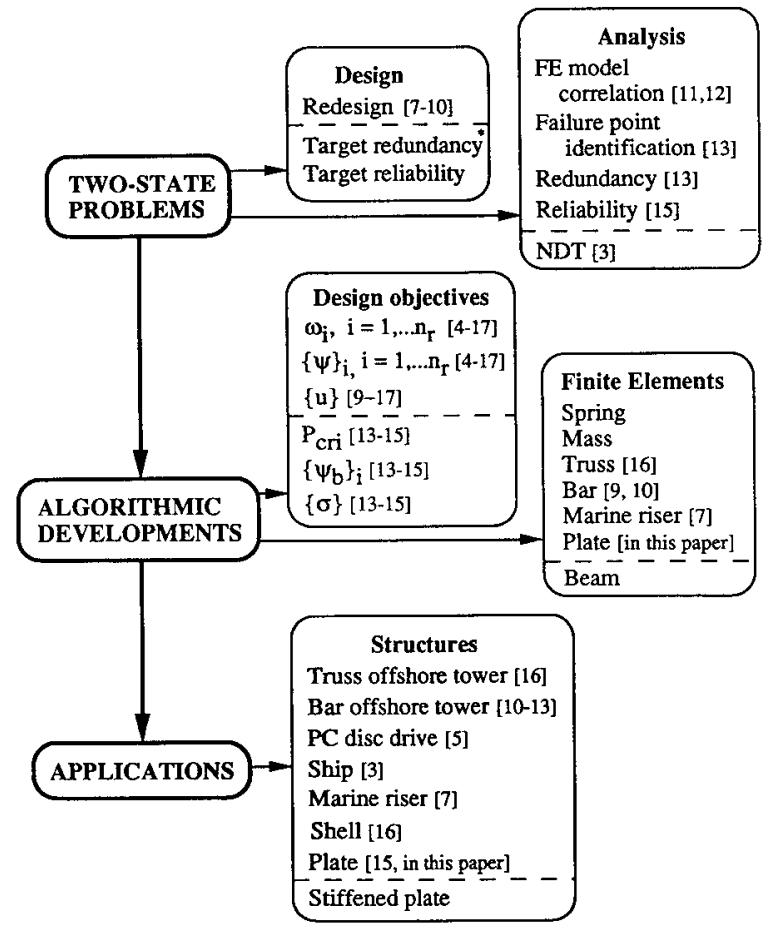

Fig. 1 Status of large admissible perturbations theory for solution of two-state problems in structural analysis and design.
Received Nov. 20, 1992; revision received July 23, 1993; accepted for publication Aug. 14, 1993. Copyright (C) 1994 by the American Institute of Aeronautics and Astronautics, Inc. All rights reserved.

*Professor, Department of Naval Architecture and Marine Engineering, North Campus. Member AIAA.

tGraduate Research Assistant, Department of Naval Architecture and Marine Engineering, North Campus. 
problems. ${ }^{15}$ State $S 1$ is the initial known design for which the required finite element analysis has been performed. State $S 2$ is the unknown design which must satisfy certain response requirements. Structural analysis problems that have been formulated and solved by nonlinear perturbation methods and in particular by the large admissible perturbations theory are: finite element (FE) model correlation, ${ }^{11-14}$ failure point identification, ${ }^{15}$ redundancy, ${ }^{15}$ and reliability. ${ }^{18}$ The nondestructive testing problem has been addressed by linear perturbation methods only. ${ }^{3}$

The problem of structural redesign (inverse design) ${ }^{7-10}$ has been formulated and solved by the large admissible perturbations theory. The problems of target redundancy and target reliability design have been formulated as two-state problems ${ }^{18}$; however, the corresponding solution algorithm has not been developed as yet.

The third goal of nonlinear perturbation methods is to develop algorithms to achieve State $S 2$ for new response requirements. Algorithms for natural frequency, ${ }^{4-19}$ normal mode, ${ }^{4-19}$ and static deflection $^{9-19}$ objectives have been developed and thoroughly tested using numerous finite element models. Critical buckling load, global buckling mode, and stress objectives have been developed ${ }^{15-17}$ recently; more applications are required, however, to fully test all aspects of related algorithms.

Algorithmic developments are also required to introduce new finite elements. Each element requires algorithmic development because the explicit dependence of the general perturbation equations on the redesign variables changes. For plates, this relation is nonlinear. The nature of the implicit dependence of those equations on the design variables does not change. This dependence can be seen in the general perturbation equations (14-17). The algorithms developed so far are to postprocess spring, mass, truss, ${ }^{18}$ bar, ${ }^{9,10}$ and marine riser ${ }^{7}$ finite elements. The plate element algorithm is developed in this paper. The most challenging research issue is to develop algorithms that allow for higher differences between baseline and objective states in redesign variables and response objectives. In our line of work,,$^{10-12,15-19}$ this goal is pursued by developing large admissible perturbations (LEAP) algorithms which make it possible to redesign a complex structure for differences of the order of $100 \%$ in redesign variables and response without repeated FEAs.

The last goal of nonlinear perturbation methods is to redesign more complex structures in terms of number of degrees of freedom, type of finite elements (beam, plate, etc.), and multiple response objectives.

In redesign, the question of uniqueness arises. In general, redesign goals can be achieved by an infinite number of different structures. Thus, an optimization criterion is needed to select the best redesign. In that respect, structural perturbation methods are related to structural optimization. Gans and Anderson ${ }^{20}$ used incremental perturbations to find an optimal turbine blade design. In each increment, a finite element run was performed at the end of the prediction and correction phases. Structural perturbation methods differ from design sensitivity methods ${ }^{21-23}$ in two ways. First, sensitivity derivatives need not to be computed, and second, the general perturbation equations provide equations for the general response of the objective state $S 2$.

The problem of plate optimization is somewhat related to the plate redesign problem studied in this paper. Several analytical ${ }^{25}$ and numerical finite element techniques ${ }^{22,23,26-31}$ have been developed to produce an optimal plate of variable thickness. Prasad and Haftka $^{26}$ minimized the weight of a plate subject to stress and displacement constraints. Good reviews of the plate optimization problem are provided in Refs. 27 and 28 . Koski et al. ${ }^{29}$ found a Pareto optimum minimizing the weight of a plate subject to stress, displacement, and frequency constraints. Natake ${ }^{30}$ used a biquadratic approximation of constraint frequencies with respect to design the variables to avoid iterative solution. $\mathrm{Lin}$ and $\mathrm{Liu}^{31}$ optimized a plate subject to buckling constraints. Sensitivity methods for optimization of plates have been used by Brockman and Lung, ${ }^{22}$ and Vanderplaats et al. ${ }^{23}$ The basic differences between other techniques and the large admissible perturbations theory are the following: 1) the latter can calculate the objective state $S 2$ for large differences between structural states $S 1$ and $S 2 ; 2$ ) no repeated FEAs are needed- $S 2$ is produced by postprocessing the FEA results of $S 1$ and the required response of state $S 2$; and 3 ) the perturbation approach to redesign (PAR) produces equations for required responses of the objective state $S 2$.

In this paper, the problem of structural redesign of plates to achieve modal dynamics and static deflection objectives is studied. The problem is formulated in Sec. II.A using PAR. A LEAP algorithm is developed in Sec. II.B. Several numerical applications for plate redesign are presented in Sec. III for single or multiple response objectives. The capability and accuracy of the large admissible perturbations theory to solve the plate redesign problem for large changes and compatible or incompatible requirements is investigated for a variable number of extracted modes and redesign variables. Results show that the new algorithm is far superior to the previous algorithm. ${ }^{9}$ The contributions of this paper consist of formulation of the plate redesign problem where the explicit—as well as the implicit-dependence of the general perturbation equations [see Eqs. (14-17)] on the redesign variables is nonlinear; and development of the corresponding LEAP algorithm for plates.

\section{Structural Redesign of Plates}

The large admissible perturbations approach to redesign consists of two parts: PAR, the perturbation approach to redesign which formulates the redesign problem as a two-state problem; and LEAP, the large admissible perturbations algorithm which calculates state $S 2$ from its required response and FEA results of state $S 1$ for structural redesign of plates. PAR is described in Sec. II.A, and LEAP in Sec. II.B.

\section{A. Perturbation Approach to Redesign}

In this paper, the general perturbation equations for modal dynamics and static deflection redesign of plates are derived and used in numerical applications. Counterpart equations for other elements, ${ }^{10-12}$ and for different response requirements (buckling and stresses ${ }^{15}$ ) can be derived. The free vibration equations for state $S 1$ are

$$
\left([k]-\omega_{j}^{2}[m]\right)\{\Psi\}_{j}=\{0\} \text { for } j=1,2, \ldots, n
$$

where the $n$ eigenvalues $\omega_{j}^{2}, j=1,2, \ldots, n$ satisfy equation

$$
\operatorname{det}\left([k]-\omega_{j}^{2}[m]\right)=0
$$

In Eq. (1), damping may be included only in Rayleigh's form. The uncoupled modal equations are

$$
\left[K_{\backslash}\right]=\left[M_{\backslash}\right]\left[\omega^{2} 、\right]
$$

where $\left[K_{\backslash}\right]$ and $\left[M_{\backslash}\right]$ are the generalized stiffness and mass matrices.

The governing equation for static $\mathrm{FE}$ analysis of state $S 1$ is

$$
[k]\{u\}=\{f\}
$$

\section{Structural Perturbations}

Let the counterparts of the Eqs. (1-4) for state $S 2$ be denoted by primed quantities. The following structural perturbations may be introduced. For modal dynamics

$$
\begin{gathered}
{\left[k^{\prime}\right]=[k]+[\Delta k]} \\
{\left[m^{\prime}\right]=[m]+[\Delta m]} \\
{\left[{ }^{\prime} \omega^{\prime 2} \backslash\right]=\left[{ }^{\prime} \omega^{2} 、\right]+\left[\backslash \Delta(\omega)^{2} \backslash\right]} \\
{\left[\phi^{\prime}\right]=[\phi]+[\Delta \phi]}
\end{gathered}
$$

where $[\phi]=\left[\{\psi\}_{1},\{\psi\}_{2}, \ldots,\{\psi\}_{n}\right]$ is the matrix of eigenvectors of $S 1$ and [ $\left.' \omega^{2} 、\right]$ is the diagonal matrix of the corresponding eigenvalues; and for static analysis

$$
\left\{u^{\prime}\right\}=\{u\}+\{\Delta u\}
$$




$$
\left\{f^{\prime}\right\}=\{f\}+\{\Delta f\}
$$

Prefix $\Delta$ indicates difference in quantities between $S 1$ and $S 2$. Even though Eqs. (5-10) appear to be difference relations, they also represent first-order terms in the linear perturbation method introduced by Stetson ${ }^{1}$ and Stetson and Harrison. ${ }^{2}$ The term linear refers to small structural changes between states $S 1$ and $S 2$, and consequently use of first-order terms only in Stetson's perturbation series. It is correct to use the term perturbation because of the approach used to derive those equations. ${ }^{1,2}$ In the large admissible perturbations theory which is used in this paper, the $\Delta$ terms in Eqs. (5-10) are not small and the solution algorithm implements an incremental prediction-correction scheme to deal with those large $\Delta$ terms. Further, a distinct perturbation parameter does not exist any longer. It is more appropriate to consider $[\Delta k]$ and $[\Delta m]$ as perturbation matrices. Accordingly, the term perturbations instead of perturbation is used when appropriate.

\section{Plate Redesign Variables}

In plate redesign, there is only one redesign variable per finite element or group of elements, that is the plate thickness $t$. Changes in stiffness and mass are expressed in terms of plate thickness changes as

$$
[\Delta k]=\sum_{e=1}^{p}\left[\Delta k_{e}\right]=\sum_{e=1}^{p}\left[\Delta k_{e}^{b}\right]+\sum_{e=1}^{p}\left[\Delta k_{e}^{m}\right]
$$

where $\left[k_{e}\right]$ is the stiffness matrix of redesign group $e$ and may include bending, torsion, stretching, etc.; $\left[k_{e}^{b}\right],\left[k_{e}^{m}\right]$ are bending and membrane stiffness matrices of redesign group $e$. Equation (11) can be written in terms of the fractional changes $\alpha_{e}$ of the plate thickness in redesign group $e$ as

$$
[\Delta k]=\sum_{e=1}^{p}\left[k_{e}^{b}\right]\left\{\left(1+\alpha_{e}\right)^{3}-1\right\}+\sum_{e=1}^{p}\left[k_{e}^{m}\right] \alpha_{e}
$$

Similarly, $[\Delta m]$ can be expressed as

$$
[\Delta m]=\sum_{e=1}^{p}\left[m_{e}\right] \alpha_{e}
$$

\section{General Perturbation Equations}

In previous work, ${ }^{9-11}$ the general perturbation equations for redesign requirements in modal dynamics and static deflections have been derived for several different finite elements (see Fig. 1). In those cases, the counterparts of Eq. (12) were linear expressions of $\alpha_{e}$. For plate redesign, the general perturbation equations for modal dynamics are derived by combining the counterpart of Eq. (3) for state $S 2$ (primed quantities) with Eqs. (5-8), (12), and (13):

$$
\begin{aligned}
& \sum_{e=1}^{p}\left\{\left\{\psi^{\prime}\right\}_{i}^{T}\left[k_{e}^{m}\right]\left\{\psi^{\prime}\right\}_{i}\left(1+\alpha_{e}\right)+\left\{\psi^{\prime}\right\}_{i}^{T}\left[k_{e}^{b}\right]\left\{\psi^{\prime}\right\}_{i}\left(1+\alpha_{e}\right)^{3}\right. \\
& \left.-\omega_{i}^{\prime 2}\left\{\psi^{\prime}\right\}_{i}^{T}\left[m_{e}\right]\left\{\psi^{\prime}\right\}_{i}\left(1+\alpha_{e}\right)\right\} \\
& =\left\{\psi^{\prime}\right\}_{i}^{T}[m]\left\{\psi^{\prime}\right\}_{i} \omega_{i}^{\prime 2}-\left\{\psi^{\prime}\right\}_{i}^{T}[k]\left\{\psi^{\prime}\right\}_{i} \\
& +\sum_{e=1}^{p}\left\{\left\{\psi^{\prime}\right\}_{i}^{T}\left[k_{e}\right]\left\{\psi^{\prime}\right\}_{i}-\omega_{i}^{\prime 2}\left\{\psi^{\prime}\right\}_{i}^{T}\left[m_{e}\right]\left\{\psi^{\prime}\right\}_{i}\right\} \\
& \quad \text { for } i=1,2, \ldots, n \\
& \sum_{e=1}^{p}\left\{\left\{\psi^{\prime}\right\}_{j}^{T}\left[k_{e}^{m}\right]\left\{\psi^{\prime}\right\}_{i}\left(1+\alpha_{e}\right)+\left\{\psi^{\prime}\right\}_{j}^{T}\left[k_{e}^{b}\right]\left\{\psi^{\prime}\right\}_{i}\left(1+\alpha_{e}\right)^{3}\right\} \\
& \quad=-\left\{\psi^{\prime}\right\}_{j}^{T}[k]\left\{\psi^{\prime}\right\}_{i}+\sum_{e=1}^{p}\left\{\psi^{\prime}\right\}_{j}^{T}\left[k_{e}\right]\left\{\psi^{\prime}\right\}_{i}
\end{aligned}
$$

$$
\begin{aligned}
& \sum_{e=1}^{p}\left\{\psi^{\prime}\right\}_{j}^{T}\left[m_{e}\right]\left\{\psi^{\prime}\right\}_{i}\left(1+\alpha_{e}\right)=-\left\{\psi^{\prime}\right\}_{j}^{T}[m]\left\{\psi^{\prime}\right\}_{i} \\
& \quad+\sum_{e=1}^{p}\left\{\psi^{\prime}\right\}_{j}^{T}\left[m_{e}\right]\left\{\psi^{\prime}\right\}_{i} \\
& \quad \text { for } \quad i=1,2, \ldots, n, \quad j=i+1, \ldots, n
\end{aligned}
$$

Equation (14) represents the Rayleigh quotients for $\omega_{i}^{\prime 2}, i=1,2$, $\ldots, n$, that is the $n$ diagonal terms of the counterpart of Eq. (3) for $S 2$. Equations (15) and (16) represent the orthogonality conditions of unknown mode $\left\{\psi^{\prime}\right\}_{i}, i=1,2, \ldots, n$, of $S 2$ with respect to [ $\left.k^{\prime}\right]$ and $\left[m^{\prime}\right]$. Theoretically, orthogonality of modes with respect to one of $[k]$ or $\left[m^{\prime}\right]$ satisfies orthogonality with respect to the other as well. Numerically, however, both conditions must be forced for $\left\{\psi^{\prime}\right\}_{i}$ to be real modes of the unknown state $S 2$.

For static redesign, the general perturbation equations are derived by combining the counterpart of Eq. (4) for state $S 2$ with Eqs. (5) and (7-10):

$$
u_{i}^{\prime}=\sum_{j=1}^{n_{r}} \frac{\phi_{i j}^{\prime}}{K_{j}^{\prime}}\left(\sum_{\ell=1}^{n} \phi_{j \ell}^{\prime} f_{\ell}\right)
$$

where

$$
K_{j}^{\prime}=\left\{\psi^{\prime}\right\}_{j}^{T}[k]\left\{\psi^{\prime}\right\}_{j}+\sum_{e=1}^{p_{p}}\left\{\left\{\psi^{\prime}\right\}_{j}^{T}\left[k_{e}^{m}\right]\left\{\psi^{\prime}\right\}_{j}\left(1+\alpha_{e}\right)\right.
$$

$$
\left.+\left\{\psi^{\prime}\right\}_{j}^{T}\left[k_{e}^{b}\right]\left\{\psi^{\prime}\right\}_{j}\left(1+\alpha_{e}\right)^{3}-\left\{\psi^{\prime}\right\}_{j}^{T}\left[k_{e}\right]\left\{\psi^{\prime}\right\}\right\}
$$

for $i=1,2, \ldots, n_{u}, \quad j=i+2, \ldots, n$

Equation (17) is derived by performing a series expansion of the static degrees of freedom $\left\{u^{\prime}\right\}$ of the unknown state $S 2$ in terms of the unknown modes $\left\{\psi^{\prime}\right\}_{i}$ of state $S 2 .^{9}$ Thus, inversion of $\left[k^{\prime}\right]$ in the counterpart of Eq. (4) for $S 2$ is avoided.

When certain response characteristics of $S 2$ are defined-natural frequencies, complete or incomplete mode shapes, or static deflections-the general perturbation equations provide the corresponding equations. Those equations depend both explicitly and implicitly on the redesign variables $\alpha_{e}$. The explicit dependence on $\alpha_{e}$ is obviously cubic. The implicit dependence is due to the dependence of the unknown modes $\left\{\psi^{\prime}\right\}_{i} i=1,2, \ldots, n$ of the objective state on the redesign variables $\alpha_{e}$. General perturbation equations for stress, critical buckling loads, and buckling modes were derived in Ref. 15 for elements other than plates. Plate redesign for stress and buckling constraints will be pursued in future work. The mathematical problem of redesign for natural frequencies and static deflections is formulated next.

\section{Problem Formulation}

Depending on the number of redesign variables $\alpha_{e}$, the number of state $S 2$ response specifications, and the number of orthogonality conditions used, the problem may be overdetermined or underdetermined. In the former case, the computer code returns a minimum error solution (see Sec. II.B). When the problem is underdetermined, solution is not unique and an optimization criterion is needed. The minimum change criterion ${ }^{3}$ given by Eq. (18) is used in our work. . $^{72,15-19}$ Other criteria, such as minimum weight or minimum static strain energy could be used. Then, the plate redesign problem can be cast in the following optimization format:

$$
\operatorname{Minimize} \sum_{e=1}^{p} \alpha_{e}^{2}
$$


subject to $n_{\omega}$ natural frequency requirements

$$
\omega_{i}^{\prime 2}=\omega_{i}^{2}+\Delta \omega_{i}^{2}, \quad i=1,2, \ldots, n_{\omega}
$$

$n_{\phi}$ normal mode requirements

$$
\phi_{k i}^{\prime}=\phi_{k i}+\Delta \phi_{k i}, \quad \text { number of }(k, i)=n_{\phi}
$$

$n_{u}$ static deflection requirements

$$
u_{i}^{\prime}=u_{i}+\Delta u_{i}, \quad i=1,2, \ldots, n_{u}
$$

$2 p$ lower and upper bounds on redesign variables

$$
-1<_{\ell} \alpha_{e}^{-} \leq_{\imath} \alpha_{e} \leq_{\ell} \alpha_{e}^{+}, \quad e=1,2, \ldots, p
$$

and $n_{a}$ orthogonality constraints [Eqs. (15) and (16)]

$$
n_{a}=2 \sum_{i=1}^{n_{\omega}}\left(n_{r}-i\right)=n_{\omega}\left[\left(2 n_{r}-1\right)-n_{\omega}\right]
$$

When the left-hand sides of Eqs. (19-21) are replaced by general perturbation equations for the specified response requirements $\Delta \omega_{i}^{2}, \Delta \phi_{k i}$, and $\Delta u_{i}$ of state $S 2$, the problem becomes strongly nonlinear in the $\alpha_{e}$ 's, both implicitly and explicitly. Thus, a solution algorithm (LEAP) must be developed to find a solution-without trial and error, or repeated FEAs-for large values of $\Delta \omega_{i}^{2}, \Delta \phi_{k i}$, $\Delta u_{i}$, and the redesign variables $\alpha_{e}$ 's.

\section{B. Large Admissible Perturbations Algorithm}

The LEAP algorithm developed to solve the plate redesign problem addresses and handles the following difficulties:

1) All required response Eqs. (14-17) and admissibility conditions for state $S 2$ depend nonlinearly on the redesign variables $\alpha_{e}$ both explicitly and implicitly. Those equations are derived by combining specification Eqs. (19-21) with the corresponding general perturbation Eqs. (14-17). The admissibility conditions used in the solution process are Eqs. (15) and (16) for which the corresponding natural frequencies have been defined.

2) The set of response objectives provided for $S 2$ is in general incomplete. That is, only a few natural frequencies, some incomplete normal modes, and some static deflections may be provided by a designer. Actually, it is practically impossible for the designer to have complete and nonconflicting response specifications for state $S 2$ because the latter is unknown.

3) State $S 2$ must be calculated by postprocessing FEA results for state $S 1$ and the specified response of state $S 2$ without repeated FEAs, trial and error, or sensitivity analysis.

The LEAP algorithm developed for plate redesign is outlined in Fig. 2. Its basic features are the following:

1) State $S 2$ is reached incrementally by changing state $S 1$ response in increments no larger than $7 \%$. In each increment, a linear inadmissible prediction and a nonlinear admissible correction are performed.

2) In the prediction phase, the increments of less than $7 \%$ are small enough to ensure that small perturbation techniques ${ }^{1-4}$ produce reasonable though inadmissible results. Only the implicit dependence on $\alpha_{e}$ 's is linearized for modal dynamics. The explicit cubic dependence is too important for plates and is not linearized. The static redesign equations are not linearized either. The prediction phase is described in Sec. II.B.1.

3) In the correction phase, prediction results are used and then corrected to satisfy the complete nonlinear equations for required response and admissibility conditions. This part of the algorithm is described in Sec. II.B.3

4) The resulting problem - in both the prediction and the correction phase - at each increment is solved by nonlinear optimization if it is underdetermined, or a minimum error algorithm if it is overdetermined. Solvers and computer implementation are described in Sec. II.B.4.

\section{Inadmissible Predictions}

For small increments, a linear perturbation technique decouples the diagonal from the off-diagonal terms of the counterpart of Eq. (3) for state $S 2$ and produces the following equations, respectively

$$
\begin{aligned}
& \{\psi\}_{i}^{T}[\delta k]\{\psi\}_{i}-\omega_{i}^{2}\{\psi\}_{i}^{T}[\delta m]\{\psi\}_{i}=M_{i} \delta \omega_{i}^{2} \\
& \text { for } i=1,2, \ldots, n_{\omega} \\
& \{\psi\}_{j}^{T}[\delta k]\{\psi\}_{i}-\omega_{i}^{2}\{\psi\}_{j}^{T}[\delta m]\{\psi\}_{i}=M_{i} c_{i j}\left(\omega_{i}^{2}-\omega_{j}^{2}\right) \\
& \text { for } i, j=1,2, \ldots, n_{r}
\end{aligned}
$$

where $M_{j}=\{\psi\}_{j}^{T}[m]\{\psi\}_{j}, n_{r}$ is the number of extracted nodes for adequate representation of state $S 2$, and $\delta$ indicates small incremental changes to distinguish them from the large global changes designated by $\Delta$. The admixture coefficients $c_{i j}$ 's are defined by

$$
[\delta \phi]=[\phi][c]^{T}
$$

where $c_{i i}=0$ and $c_{i j}$ 's are small.

For plate redesign, Eqs. (24) and (25) become

$$
\begin{aligned}
& \sum_{e=1}^{p}\left\{\left(\{\psi\}_{i}^{T}\left[k_{e}^{m}\right]\{\psi\}_{i}-\omega_{i}^{2}\{\psi\}_{i}^{T}\left[m_{e}\right]\{\psi\}_{i}\right)\left(1+\alpha_{e}\right)\right. \\
& \left.+\{\psi\}_{i}^{T}\left[k_{e}^{b}\right]\{\psi\}_{i}\left(1+\alpha_{e}\right)^{3}\right\} \\
& =M_{i} \delta \omega_{i}^{2}+\sum_{e=1}^{p}\left(\{\psi\}_{i}^{T}\left[k_{e}\right]\{\psi\}_{i}-\omega_{i}^{2}\{\psi\}_{i}^{T}\left[m_{e}\right]\{\psi\}_{i}\right) \\
& i=1,2, \ldots, n_{\omega}
\end{aligned}
$$

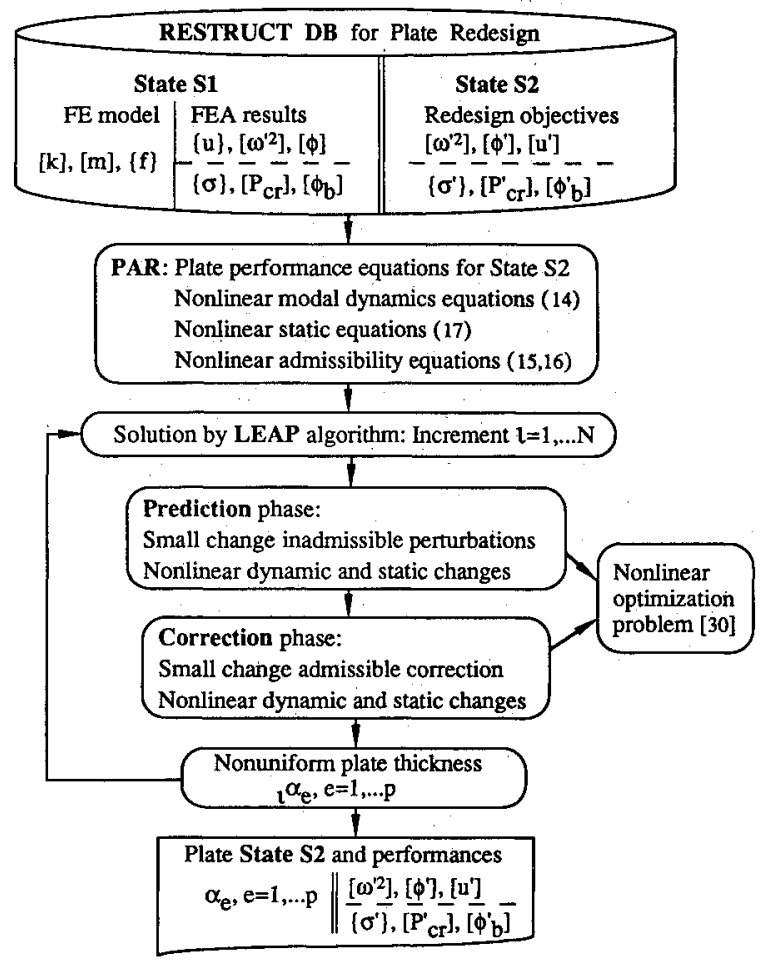

Fig. 2 Plate redesign by a large admissible perturbations algorithm. 


$$
\begin{aligned}
& \sum_{e=1}^{p}\left\{\{\psi\}_{j}^{T}\left[k_{e}^{m}\right]\{\psi\}_{i}\left(1+\alpha_{e}\right)+\{\psi\}_{i}^{T}\left[k_{e}^{b}\right]\{\psi\}_{i}\left(1+\alpha_{e}\right)^{3}\right. \\
& \left.-\omega_{i}^{2}\{\psi\}_{i}^{T}\left[m_{e}\right]\{\psi\}_{i}\left(1+\alpha_{e}\right)\right\}=M_{j} c_{i j}\left(\omega_{i}^{2}-\omega_{j}^{2}\right) \\
& +\sum_{e=1}^{p}\left(\{\psi\}_{j}^{T}\left[k_{e}\right]\{\psi\}_{i}-\omega_{i}^{2}\{\psi\}_{i}^{T}\left[m_{e}\right]\{\psi\}_{i}\right) \\
& i_{j}=1,2, \ldots, n_{r}
\end{aligned}
$$

The $n_{u}$ static requirement equations defined by Eqs. (17) and (21) are used as they are in their complete nonlinear form.

In the prediction phase in increment $\ell, \ell=1,2, \ldots, N$, the problem defined in Sec. II.A. 4 reduces to the following problem:

$$
\min \sum_{e=1}^{p}\left[\left(1+\alpha_{e}\right) \prod_{q=1}^{\ell-1}\left(1+{ }_{q} \alpha_{e}\right)-1\right]^{2}
$$

subject to $n_{\omega}$ frequency requirements (27), $n_{\mathrm{\phi}}$ modal requirements defined by Eqs. (20) and (28), $n_{u}$ static requirements (17), and $2 p$ lower and upper bounds on the redesign variables

$$
-1<{ }_{\ell} \alpha_{e}^{-} \leq{ }_{\ell} \alpha_{e} \leq{ }_{\ell} \alpha_{e}^{+}, \quad e=1,2, \ldots, p
$$

This problem is nonlinear and solution is achieved using the nonlinear optimization code NPSOL ${ }^{32}$ implemented in the redesign code. ${ }^{16}$

\section{Cognate Space}

At the end of the prediction phase, the admixture coefficients $c_{i j}$, $i, j=1,2, \ldots, n_{r}$ are computed using Eq. (28). Then the normal modes that have not been specinied-as per Eq. (20)-are computed using Eq. (26). Computation of admixture coefficients may consume as much as $80 \%$ of the required CPU time in a given increment. Thus, in the first increment, the cognate space for the modes that are being changed is identified. ${ }^{11}$ Admixture coeffi-
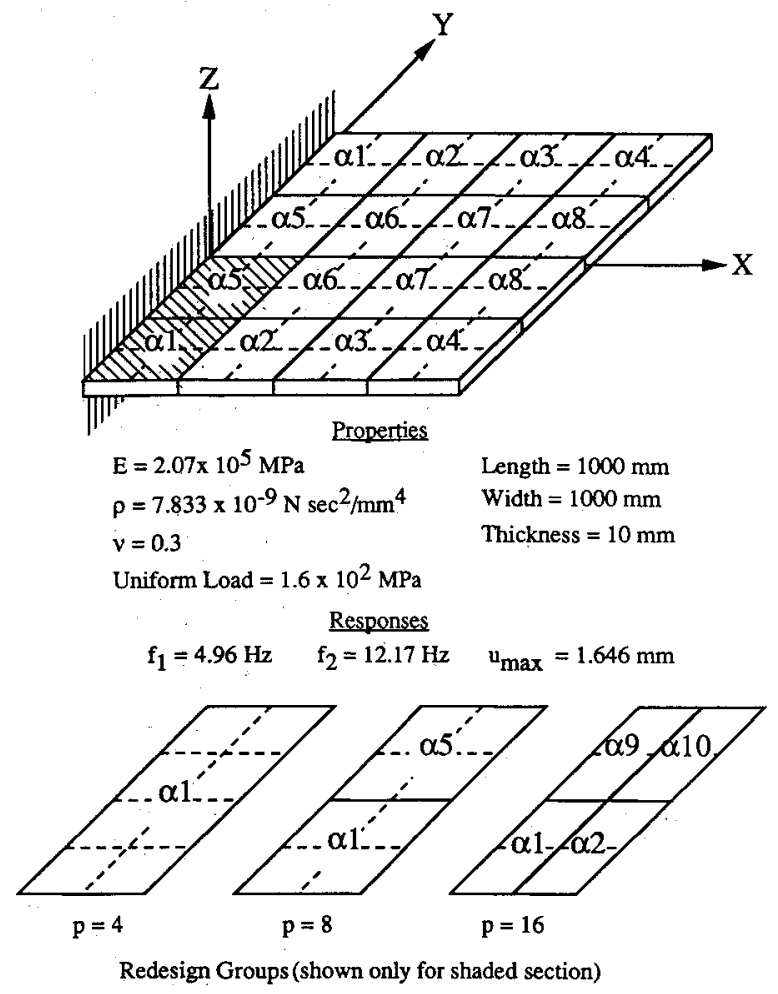

Fig. 3 Cantilever plate FE model and redesign groups. cients between modes in different cognate spaces have very small values and are set equal to zero. This significantly reduces the required CPU time. Cognate spaces are identified numerically by setting a lower limit of $10^{-6}$ on the admixture coefficient. Previous applications show that cognate spaces have physical meaning. For example, all bending modes of a structure may constitute one cognate space, torsional modes may constitute another space, etc. ${ }^{11,12,15-19}$

\section{Admissible Corrections}

At this point we could proceed to the next increment. The error could build up fast, however, unless a new FEA is used to update the modal basis. To avoid repeated FEAs the results of the prediction phase are corrected by satisfying the nonlinear specification equations (14) and (17) and the admissibility conditions (15) and (16). The specified modes and those predicted by Eq. (26) at the end of the prediction phase are used. Here, the term admissibility must be explained. The orthogonality conditions (15) and (16) have been linearized in the prediction phase to produce the admixture coefficients $c_{i j}$ [Eq. (28)]. In the correction phase, the complete nonlinear expressions (15) and (16) must be forced so that the modes computed by Eq. (26) correspond to the structure specified at the end of the correction phase by the redesign variables $\alpha_{\rho}$. Consequently, the orthogonality conditions determine whether the incremental changes are admissible (real) or not. Summarizing, the problem solved in the correction phase is the following: satisfy the optimization criterion (29) subject to $n_{\omega}$ requirements (14), $n_{u}$ requirements (17), and $n_{a}$ admissibility conditions (15) and (16). The theoretical number $n_{a}$ is given by Eq. (22). In practice, most dominant admissibility conditions are satisfied.

At the end of the correction phase, after the new incremental values of the redesign variables ${ }_{i} \alpha_{e}$ have been computed, Eq. (31) is used to compute unspecified natural frequencies of state $S 2$

$$
\omega_{i}^{2}=\frac{K_{i}^{\prime}}{M_{i}^{\prime}}=\frac{\left\{\psi^{\prime}\right\}_{i}^{T}\left[k^{\prime}\right]\left\{\psi^{\prime}\right\}_{i}}{\left\{\psi^{\prime}\right\}_{i}^{T}\left[m^{\prime}\right]\left\{\psi^{\prime}\right\}_{i}}
$$

and Eq. (17) to compute unspecified static deflections.

\section{Computer Implementation}

The LEAP algorithm described earlier is implemented in code, the program for redesign of structures (RESTRUCT) which is presently about 27,000 Fortran 77 commands and postprocesses data produced by MSC/NASTRAN on the University of Michigan main frame computer IBM 9021 . Next, several numerical applications are used to demonstrate the ability of the developed LEAP algorithm to produce accurate results for large differences between states $S 1$ and $S 2$.

\section{Numerical Applications}

A rectangular plate of thickness $t=10 \mathrm{~mm}$ and side $\ell=1000$ $\mathrm{mm}$, clamped at one edge and free along the other three, subject to a uniform load of $100 \mathrm{MPa}$ is used in all numerical redesign applications in this section (see Fig. 3). First, systematic finite element analyses were performed using MSC/NASTRAN to establish a satisfactory FE model. Figure 4 compares the first ten natural frequencies of the cantilever plate as computed using 16,64 , and 256 finite elements. An analytical expression for the first six natural frequencies provides additional data for comparison. ${ }^{33-35}$ On the basis of Fig. 4, it was decided that the 64-element model is accurate enough for the purpose of this work.

Numerous applications were run and the results are summarized in Tables 1-5. Three different redesign requirements were used in sets of one (Table 1), two (Tables 2-4), or three (Table 5). Each table, for each case, shows the case number; the redesign goals in the form of a ratio as $\omega_{1}^{\prime 2} / \omega_{1}^{2}, \omega_{2}^{\prime 2} / \omega_{2}^{2}$, and $u_{\max }^{\prime} / u_{\max }$; the percentage error in achieving the goals in comparison to reanalysis performed by MSC/NASTRAN for the redesign produced by RESTRUCT; the CPU time in seconds. It should be pointed out that implementing the complete nonlinear expression (17) for the static deflection requirements has eliminated the prediction error 


\begin{tabular}{|c|c|c|c|c|c|}
\hline \multirow{2}{*}{$\begin{array}{c}\text { MODE } \\
\#\end{array}$} & \multirow{2}{*}{$\begin{array}{l}\text { MODE } \\
\text { SHAPE }\end{array}$} & \multicolumn{4}{|c|}{ NATURAL FREQUENCIES } \\
\hline & & Analytic[32] & $16 \mathrm{FE}$ & $64 \mathrm{FE}$ & $256 \mathrm{FE}$ \\
\hline 1 & & 4.99 & 4.95 & 4.96 & 4.96 \\
\hline 2 & L- & 12.19 & 12.35 & 12.17 & 12.15 \\
\hline 3 & & 30.63 & 32.31 & 30.96 & 30.56 \\
\hline 4 & & 39.07 & 41.48 & 39.50 & 39.01 \\
\hline 5 & {$[E]$} & 44.47 & 49.19 & 45.13 & 44.40 \\
\hline 6 & & 77.82 & 90.89 & 80.12 & 77.86 \\
\hline 7 & L & & 103.63 & 92.79 & 88.91 \\
\hline 8 & $\stackrel{a}{a}$ & & 112.11 & 96.96 & 92.93 \\
\hline 9 & 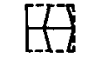 & & 138.63 & 108.67 & 102.96 \\
\hline 10 & 乎 & & 173.45 & 142.15 & 134.59 \\
\hline
\end{tabular}

Fig. 4 Natural frequencies and mode shapes of cantilever plate.

in the redesign algorithm which was computed in previous publications. ${ }^{10-17}$ Tables $1-5$ also provide information about the number of redesign variables $p$, the number of extracted modes $n_{r}$, and the number of admissibility constraints $n_{a}$. Table 6 shows the optimal values of the redesign variables $\alpha_{e}, e=1,2, \ldots, p$ for five different applications with compatible objectives. Table 7 shows similar data for five more applications.

\section{A. Single Redesign Requirements}

This is the simplest of all redesign problems. Eight cases are shown in Table 1 and the error is trivial even for changes by a factor of two in either redesign goal $\omega_{1}^{\prime 2} / \omega_{1}^{2}$ or $u_{\max }^{\prime} / u_{\max }$. Higher changes in redesign goals, by a factor of three or more, can be achieved without additional FEAs and with very small prediction error.

\section{B. Two Compatible Redesign Requirements}

In Table 2, redesign applications for two simultaneous requirements $\omega_{1}^{\prime 2} / \omega_{1}^{2}$ and $u_{\max }^{\prime} / u_{\max }$ are shown. Redesign objectives in this table are labeled compatible; and this term needs clarification. We call compatible redesign goals those that would cause similar changes in a uniform plate. This is by no means a rigorous definition. The following examples are helpful in clarifying the term. Doubling the first natural frequency increases the bending rigidity of the plate; reducing the maximum static deflection by a factor of two has similar effect; such changes are labeled compatible. As a second example, consider doubling the first natural frequency and the maximum deflection. Such redesign objectives are labeled incompatible. In nonuniform plate redesign, as are all the cases in Tables 1-6, incompatible requirements result in more dramatic changes in the thickness of plate groups. As a third example, consider the case of $\left(\omega_{1}^{\prime 2} / \omega_{1}^{2}=2.0, u_{\max }^{\prime} / u_{\max }=0.8\right)$; even though an analytically compatible change would require $u_{\max }^{\prime} / u_{\max }=0.5$, the change of $(2.0,0.8)$ is still labeled compatible.

Twelve cases of compatible redesign requirements are shown in Table 2 . The error is very small even for changes by factor of two in the redesign requirements. By comparing cases 22-1-22-4 and 231-23-4 we observe that the error increases as the redesign objective values depart from the ideal uniform plate relation $\left(\omega_{1}^{2} / \omega_{1}^{2}\right) \times$ $\left(u_{\max }^{\prime} / u_{\max }\right)=1$. Comparing cases $11-4,12-4$ and $22-4$, we observe that the optimal redesigns are practically identical as shown in Table 6.
Table 4 shows six more cases (41 and 44) of compatible redesign goals in the first and second eigenvalues. Results are significantly more accurate than in the case of incompatible objectives as in cases 42 and 43 .

\section{Two Incompatible Redesign Requirements}

Twelve redesign applications with incompatible redesign objectives are shown in Table 3 . Ten more cases 42,43 are shown in Table 4 . Table 3 shows that the error increases very fast. This is so because dramatic departure from the uniform plate redesign is required to achieve such highly incompatible changes where $\left(\omega_{1}^{\prime 2} /\right.$ $\left.\omega_{1}^{2}\right) \times\left(u_{\max }^{\prime} / u_{\max }\right)=4.0$. There are two ways in which the redesign results can be improved. In cases 31-6 and 32-6, the incremental changes in redesign goals were reduced from 7 to $4 \%$; this, however, results in small improvement. In cases 31-5 and 32-5, an

Table 1 Plate redesign for single requirement

\begin{tabular}{|c|c|c|c|c|c|}
\hline \multirow[b]{2}{*}{ Case $^{\mathrm{a}} \#$} & \multicolumn{2}{|c|}{ Goals } & \multicolumn{2}{|c|}{ Error $(\%)^{b}$} & \multirow[b]{2}{*}{$\mathrm{CPU}(\mathrm{s})$} \\
\hline & $\omega_{1}^{\prime 2} / \omega_{1}^{2}$ & $u_{\max }^{\prime} / u_{\max }$ & $\omega_{1}^{\prime 2} / \omega_{1}^{2}$ & $u_{\max }^{\prime} / u_{\max }$ & \\
\hline $11-1$ & 1.065 & $=$ & 0.014 & - & 56 \\
\hline $11-2$ & 1.287 & - & 0.177 & - & 210 \\
\hline $11-3$ & 1.460 & - & 0.354 & - & 313 \\
\hline $11-4$ & 2.000 & - & 0.917 & - & 569 \\
\hline $12-1$ & - & 0.933 & - & -0.004 & 64 \\
\hline $12-2$ & - & 0.758 & - & -0.182 & 249 \\
\hline $12-3$ & $\ldots$ & 0.660 & - & -0.381 & 371 \\
\hline $12-4$ & - & 0.500 & - & -0.831 & 616 \\
\hline
\end{tabular}

${ }^{\mathrm{a}}$ In all cases, $p=8, n_{r}=5$, and $n_{a}=4$. bError $(\%)=100 \times($ goal - reanalysis $) /$ reanal ysis.

Table 2 Plate redesign for $\omega_{1}^{\prime 2}$ and $u_{\max }^{\prime}$ : compatible requirements

\begin{tabular}{lccccccc}
\hline Case $^{\mathrm{a}}$ & \multicolumn{2}{c}{ Goals } & \multicolumn{3}{c}{ Error $(\%)^{\mathrm{b}}$} & CPU & \\
\cline { 2 - 7 }$\#$ & $\omega_{1}^{\prime 2} / \omega_{1}^{2}$ & $u_{\max }^{\prime} / u_{\max }$ & $\omega_{1}^{\prime 2} / \omega_{1}^{2}$ & $u_{\max }^{\prime} / u_{\max }$ & $(\mathrm{s})$ & $n_{r}$ & $n_{a}$ \\
\hline $21-1$ & 1.065 & 0.939 & 0.017 & -0.071 & 39 & 3 & 2 \\
$21-2$ & 1.287 & 0.777 & 0.212 & -0.248 & 143 & 3 & 2 \\
$21-3$ & 1.459 & 0.685 & 0.420 & -0.478 & 212 & 3 & 2 \\
$21-4$ & 2.000 & 0.500 & 1.098 & -1.387 & 383 & 3 & 2 \\
$22-1$ & 1.065 & 0.939 & 0.015 & -0.001 & 73 & 5 & 4 \\
$22-2$ & 1.287 & 0.777 & 0.199 & -0.155 & 276 & 5 & 4 \\
$22-3$ & 1.459 & 0.685 & 0.398 & -0.323 & 411 & 5 & 4 \\
$22-4$ & 2.000 & 0.500 & 1.012 & -0.836 & 748 & 5 & 4 \\
$23-1$ & 1.065 & 0.980 & 0.008 & 0.006 & 74 & 5 & 4 \\
$23-2$ & 1.287 & 0.922 & 0.134 & -0.134 & 278 & 5 & 4 \\
$23-3$ & 1.460 & 0.885 & 0.312 & -0.376 & 414 & 5 & 4 \\
$23-4$ & 2.000 & 0.800 & 1.238 & -2.104 & 748 & 5 & 4 \\
\hline \hline
\end{tabular}

${ }^{\mathrm{a}}$ In all cases, $p=8$. bError $(\%)=100 \times($ goal - reanalysis $) /$ reanalysis.

Table 3 Plate redesign for $\omega_{1}^{\prime 2}$ and $u_{\max }^{\prime}$ : incompatible requirements

\begin{tabular}{lcccccccc}
\hline \hline Case $^{\mathrm{a}}$ & \multicolumn{2}{c}{ Goals } & \multicolumn{5}{c}{ Error $(\%)^{\mathrm{b}}$} & \multicolumn{2}{c}{ CPU } \\
\cline { 2 - 7 } & $\omega_{1}^{\prime 2} / \omega_{1}^{2}$ & $u_{\max }^{\prime} / u_{\max }$ & $\omega_{1}^{\prime 2} / \omega_{1}^{2}$ & $u_{\max }^{\prime} / u_{\max }$ & $(\mathrm{s})$ & $n_{r}$ & $n_{a}$ \\
\hline $31-1$ & 1.065 & 1.065 & 0.038 & -0.201 & 40 & 3 & 2 \\
$31-2$ & 1.287 & 1.287 & 1.070 & -2.478 & 143 & 3 & 2 \\
$31-3$ & 1.459 & 1.459 & 3.427 & -7.254 & 212 & 3 & 2 \\
$31-4$ & 2.000 & 2.000 & 28.25 & -41.33 & 384 & 3 & 2 \\
$31-5^{\mathrm{c}}$ & 2.000 & 2.000 & 6.764 & -18.35 & 426 & 3 & 2 \\
$31-6^{\mathrm{d}}$ & 1.080 & 1.080 & 0.097 & -0.376 & 75 & 3 & 2 \\
$31-7^{\mathrm{d}}$ & 1.470 & 1.470 & 2.799 & -8.572 & 354 & 3 & 2 \\
$32-1$ & 1.065 & 1.065 & 0.031 & -0.017 & 74 & 5 & 4 \\
$32-2$ & 1.287 & 1.287 & 0.868 & -1.131 & 276 & 5 & 4 \\
$32-3$ & 1.459 & 1.459 & 2.919 & -4.032 & 411 & 5 & 4 \\
$32-4$ & 2.000 & 2.000 & 30.44 & -30.92 & 748 & 5 & 4 \\
$32-5^{\mathrm{c}}$ & 2.000 & 2.000 & 6.571 & -15.14 & 752 & 5 & 4 \\
\hline \hline
\end{tabular}

an all cases, $p=8$

${ }^{b}$ Error $(\%)=100 \times($ goal - reanalysis $) /$ reanalysis.

cState $S 1$ in cases \#31-5 and \#32-5 are produced by an intermediate FEA from cases \#31-3 and \#32-3, respectively.

$\mathrm{d} 4 \%$ increments, $7 \%$ in all other cases. 
Table 4 Plate redesign for $\omega_{1}^{\prime 2}$ and $\omega_{2}^{\prime 2}$ : compatible and incompatible requirements

\begin{tabular}{lccccc}
\hline \hline & \multicolumn{2}{c}{ Goals } & \multicolumn{2}{c}{ Error $(\%)^{b}$} & \\
\cline { 2 - 4 } Case $^{\mathrm{a}} \#$ & $\omega_{1}^{\prime 2} / \omega_{1}^{2}$ & $\omega_{2}^{\prime 2} / \omega_{2}^{2}$ & $\omega_{1}^{\prime 2} / \omega_{1}^{2}$ & $\omega_{2}^{\prime 2} / \omega_{2}^{2}$ & CPU (s) \\
\hline $41-1$ & 1.065 & 1.065 & 0.014 & 0.039 & 75 \\
$41-2$ & 1.460 & 1.460 & 0.420 & 1.382 & 427 \\
$41-3$ & 2.000 & 2.000 & 1.211 & 4.332 & 781 \\
$42-1$ & 1.065 & 0.939 & 0.342 & 0.349 & 76 \\
$42-2$ & 1.208 & 0.828 & 2.910 & 2.516 & 220 \\
$42-3$ & 1.459 & 0.658 & 10.91 & 11.21 & 434 \\
$42-4^{\mathrm{c}}$ & 1.459 & 0.658 & 1.704 & 4.323 & 492 \\
$42-5^{\mathrm{d}}$ & 1.212 & 0.825 & 2.528 & 2.334 & 362 \\
$42-6^{\mathrm{d}}$ & 1.470 & 0.680 & 8.579 & 12.19 & 718 \\
$43-1$ & 0.939 & 1.065 & 0.195 & 0.104 & 77 \\
$43-2$ & 0.828 & 1.208 & 2.123 & 1.037 & 220 \\
$43-3$ & 0.685 & 1.460 & 10.54 & 9.508 & 435 \\
$43-4^{\mathrm{c}}$ & 0.685 & 1.460 & 1.636 & 1.669 & 442 \\
$43-5^{\mathrm{e}}$ & 0.680 & 1.470 & 9.370 & 8.684 & 714 \\
$44-1$ & 0.933 & 0.933 & 0.012 & 0.046 & 78 \\
$44-2$ & 0.660 & 0.660 & 0.556 & 1.530 & 439 \\
$44-3$ & 0.500 & 0.500 & 1.586 & 3.925 & 726 \\
\hline \hline
\end{tabular}

${ }^{\mathrm{a}}$ In all cases, $p=8, n_{r}=5$, and $n_{a}=5$.

${ }^{b}$ Error $(\%)=100 \times($ goal - reanalysis $) /$ reanalysis.

cBaseline is case \#42-3.

$\mathrm{d} 4 \%$ increment, $7 \%$ in all other cases.

Baseline is case \#43-3.

Table 5 Redesign for $\omega_{1}^{2}, \omega_{2}^{2}$, and $u_{\max }$

\begin{tabular}{|c|c|c|c|c|c|c|c|c|}
\hline \multirow{2}{*}{$\begin{array}{l}\text { Case }^{\mathrm{a}} \\
\#\end{array}$} & \multicolumn{3}{|c|}{ Goals } & \multicolumn{3}{|c|}{ Error $(\%)^{b}$} & \multirow{2}{*}{\multicolumn{2}{|c|}{$\begin{array}{ll} & \mathrm{CPU} \\
p & (\mathrm{~s})\end{array}$}} \\
\hline & $\omega_{1}^{\prime 2} / \omega_{1}^{2}$ & $\omega_{2}^{\prime 2} / \omega_{2}^{2}$ & $u_{\max }^{\prime} / u_{\max }$ & $\omega_{1}^{\prime 2} / \omega_{1}^{2}$ & $\omega_{2}^{\prime 2} /$ & $u_{\max }^{\prime} / u_{\max }$ & & \\
\hline 51-1 & 1.459 & 1.459 & 0.685 & 1.115 & 2.239 & -1.169 & 4 & 463 \\
\hline $1-2$ & 2.000 & 2.000 & 0.500 & 2.587 & 5.762 & -2.754 & 4 & 844 \\
\hline $2-1$ & 1.4 & 1.459 & 0 & 0.430 & 1. & -0.388 & 8 & 530 \\
\hline $2-2$ & 2.000 & 2.000 & 0.500 & 1.286 & 4. & -1.075 & 8 & 967 \\
\hline $3-1$ & 1.459 & 1.459 & 0.685 & 0.529 & 1.747 & -0.644 & 16 & 530 \\
\hline-2 & 2.000 & 2.000 & 050 & 1.001 & & -1.070 & 16 & 966 \\
\hline-1 & & 1.248 & 0 & 00 & 72 & -0 & 4 & 463 \\
\hline-2 & & 1.500 & 0 & 11 & 03 & -1 & 4 & 844 \\
\hline $5-1$ & 1.459 & 1.248 & 0.685 & 0.371 & 0.220 & -0.300 & 8 & 531 \\
\hline 5-2 & 2.000 & 1.500 & 0.500 & 0.994 & 0.387 & -0.814 & 8 & 968 \\
\hline $56-1$ & 1.459 & 1.248 & 35 & 0.123 & 0.225 & -0.053 & 16 & 531 \\
\hline$?$ & & 1.500 & 0 & 18 & 26 & -0.120 & 16 & 969 \\
\hline $7-1$ & 1.447 & 1.447 & 0.574 & 0.508 & 1.213 & -0.467 & 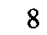 & 703 \\
\hline $77-2$ & 2.000 & 2.000 & 0.354 & 1.415 & 3.404 & -1.288 & 8 & 1314 \\
\hline $58-1$ & 1.447 & 1.241 & 0.574 & 1.402 & 1.062 & -1.276 & . & 704 \\
\hline 88-2 & 2.000 & 1.500 & 0.354 & 4.804 & 4.281 & -4.329 & 8 & 1311 \\
\hline
\end{tabular}

${ }^{\mathrm{a}}$ In all cases, $n_{r}=5$ and $n_{a}=4 . \quad{ }^{\mathrm{b}} \mathrm{Error}(\%)=100 \times($ goal - reanalysis $) /$ reanalysis.

intermediate FEA was used with incremental changes of $7 \%$. A similar observation can be made in Table 4 .

\section{Three Redesign Objectives}

Compatibility is even more difficult to define in this case. The general concept, however, of compatibility defined in Sec. III.B still holds. The tables show sixteen redesign applications with three simultaneous redesign requirements for $\omega_{1}^{\prime 2} / \omega_{1}^{2}, \omega_{2}^{\prime 2} / \omega_{2}^{2}$, and $u_{\max }^{\prime} / u_{\max }$. These requirements could be classified as compatible even though there is significant departure of requirements from analytical compatibility for uniform plate. The accuracy of computations in finding the optimal state $S 2$ without any FEA's is impressive even in case \#58-2 where $u_{\max }^{\prime} / u_{\max }$ changes by a factor of nearly three.

\section{E. Effect of Redesign Variables and Extracted Modes}

The accuracy of redesign by large admissible perturbations depends on the number of redesign variables $p$, the number of extracted modes $n_{r}$, and the number of admissibility conditions $n_{a}$ which is a function of $n_{r}$ given by Eq. (22). Increasing the number of redesign variables gives more flexibility to redesign optimiza-
Table 6 Plate redesign variables for five applications with compatible requirements

\begin{tabular}{lcccccc}
\hline Case \# & $11-4$ & $12-4$ & $22-4$ & $55-2$ & $56-2$ & \\
\hline$\alpha_{1}$ & 0.292 & 0.292 & 0.294 & 0.297 & 0.297 & $\alpha_{1}$ \\
& & & & & 0.276 & $\alpha_{2}$ \\
$\alpha_{2}$ & 0.169 & 0.170 & 0.170 & 0.170 & 0.217 & $\alpha_{3}$ \\
$\alpha_{3}$ & 0.046 & 0.046 & 0.043 & 0.048 & 0.138 & $\alpha_{4}$ \\
& & & & & 0.068 & $\alpha_{5}$ \\
$\alpha_{4}$ & 0.003 & 0.003 & -0.005 & -0.007 & -0.002 & $\alpha_{6}$ \\
& & & & & -0.009 & $\alpha_{7}$ \\
$\alpha_{5}$ & 0.375 & 0.375 & 0.375 & 0.371 & 0.414 & $\alpha_{9}$ \\
& & & & & 0.289 & $\alpha_{10}$ \\
$\alpha_{6}$ & 0.162 & 0.162 & 0.162 & 0.163 & 0.211 & $\alpha_{11}$ \\
& & & & & 0.135 & $\alpha_{12}$ \\
$\alpha_{7}$ & 0.046 & 0.046 & 0.042 & 0.039 & 0.067 & $\alpha_{13}$ \\
& & & & & 0.023 & $\alpha_{14}$ \\
$\alpha_{8}$ & 0.004 & 0.004 & -0.005 & -0.003 & 0.004 & $\alpha_{15}$ \\
& & & & & 0.000 & $\alpha_{16}$ \\
\hline \hline
\end{tabular}

Table 7 Plate redesign variables for five applications

\begin{tabular}{lcccccc}
\hline \hline Case \# & $11-3$ & $22-3$ & $32-3$ & $42-6$ & $56-1$ & \\
\hline$\alpha_{1}$ & 0.140 & 0.160 & -0.069 & -0.251 & 0.176 & $\alpha_{1}$ \\
& & & & & 0.139 & $\alpha_{2}$ \\
$\alpha_{2}$ & 0.063 & 0.078 & -0.092 & -0.159 & 0.103 & $\alpha_{3}$ \\
& & & & & 0.062 & $\alpha_{4}$ \\
$\alpha_{3}$ & -0.004 & 0.016 & -0.383 & -0.200 & 0.028 & $\alpha_{5}$ \\
& & & & & 0.007 & $\alpha_{6}$ \\
$\alpha_{4}$ & -0.063 & 0.000 & -0.568 & -0.080 & -0.003 & $\alpha_{7}$ \\
& & & & & -0.006 & $\alpha_{8}$ \\
$\alpha_{5}$ & 0.166 & 0.189 & -0.073 & 0.372 & 0.206 & $\alpha_{9}$ \\
& & & & & 0.139 & $\alpha_{10}$ \\
$\alpha_{6}$ & 0.059 & 0.072 & -0.096 & -0.151 & 0.097 & $\alpha_{11}$ \\
& & & & & 0.058 & $\alpha_{12}$ \\
$\alpha_{7}$ & -0.006 & 0.016 & -0.388 & -0.342 & 0.027 & $\alpha_{13}$ \\
$\alpha_{8}$ & -0.065 & 0.000 & -0.589 & -0.532 & 0.004 & $\alpha_{15}$ \\
& & & & & 0.006 & $\alpha_{16}$ \\
\hline \hline
\end{tabular}

tion and in general reduces the prediction error. The first twelve cases in Table 5 show the dependence of the error on the number of redesign variables.

The effect of the extracted modes and the number of admissibility conditions is shown in Tables 2 and 3. In all applications in this paper, it was found that the effect of the mass admissibility conditions was trivial and were eliminated from the redesign process. All stiffness admissibility conditions were used. In general, accuracy is improved by increasing the number of extracted modes and admissibility conditions. There is a limit, however, to the validity of the statement. Specifically, if the number of admissibility conditions is increased to the point of having more equality constraints than redesign variables in the optimization process, then the problem will be overdetermined, and there will be no solution.

\section{F. Optimal Redesigns}

Table 6 shows the plate redesign variables for five applications with compatible redesign goals. The first four cases show practically identical results. In case $11-4 \omega_{1}^{\prime 2} / \omega_{1}^{2}=2.0$; in case $12-4$, $u_{\max }^{\prime} / u_{\max }=0.5$; in case $22-4$ the previous two objectives are used simultaneously; in case $55-2$, the third redesign requirements $\omega_{2}^{\prime 2} / \omega_{2}=1.5$ is added. In the last case $56-2$, the same three simultaneous requirements as in case 55-2 are used; the number of design variables, however, is doubled. Comparison of cases 55-2 and 56-2 shows that the tendency in the redesign is identical. In case 56-2, however, variation of the plate thickness is much smoother as expected. Further, the optimal value of the objective $\Sigma_{e=1}^{p=8} \alpha_{e}^{2}$ in case $55-2$ is 0.5342 ; that multiplied by 2 , to account for the different number of redesign variables, is larger than the optimal value of $\Sigma_{e=1}^{p=16} \alpha_{e}^{2}=0.7482$ in case 56-2. 
Table 7 shows the plate redesign variables for five applications for which the redesign requirements are not ideally compatible.

\section{Closing Remarks}

Formulation of the plate redesign problem to achieve modal dynamics and static deflection requirements using the perturbation approach to redesign (PAR), and solution by a large admissible perturbations (LEAP) algorithm, has further established the capability and potential of the large admissible perturbations theory to address and solve two-state problems in structural analysis and design. Implementation of the complete nonlinear expressions of the general perturbation equations for both modal dynamics and static deflection requirements have made predictions by code RESTRUCT nearly exact and have reduced redesign errors drastically. Even in the case of redesign for multiple requirements, the accuracy of the LEAP algorithm improves with the flexibility of the model. Specifically, higher number of degrees of freedom and redesign variables improves the accuracy of the solutions. Accuracy also depends on the number of extracted modes and the number of imposed admissibility conditions. Compatibility of multiple requirements is a very important factor. Incompatibility results in excessive departure (by a factor of two or more) from the original design and reduces the accuracy in redesign solutions. In the extreme case where multiple requirements are conflicting, there is no optimal solution (to minimum change criterion) and a minimum error algorithm is used to solve the problem. The successful development of a LEAP algorithm for plate redesign has made it possible to address in the near future the problem of stiffened plate redesign. The major challenge in that problem is the shifting of the neutral axis during the redesign process.

\section{Acknowledgment}

Research support provided by the Office of Naval Research through Grant No. DOD-G-B00014-90-J-4081 is gratefully acknowledged.

\section{References}

${ }^{1}$ Stetson, K. A., "Perturbation Method of Structural Design Relevant to Holographic Vibration Analysis," AIAA Journal, Vol. 13, No. 4, 1975, pp. $457-459$.

${ }^{2}$ Stetson, K. A., and Harrison, I. R., "Redesign of Structural Vibration Modes by finite element Inverse Perturbation," ASME Transactions, Journal of Engineering for Power, Vol. 103, No. 2, 1981, pp. 319-325.

${ }^{3}$ Sandström, R. E., and Anderson, W. J., "Modal Perturbation Methods for Marine Structures," Transactions, SNAME, Vol. 90, 1982, pp. 41-54.

${ }^{4} \mathrm{Kim}, \mathrm{K}$. O., Anderson, W. J., and Sandström, R. E., "Nonlinear Inverse Perturbation Method in Dynamic Analysis," AIAA Journal, Vol. 21, No. 9, 1983, pp. 1310-1316.

${ }^{5}$ Hoff, C. J., Bernitsas, M. M., Sandström, R. E., and Anderson, W. J., "Nonlinear Incremental Inverse Perturbation Method for Structural Redesign," AlAA Journal, Vol. 22, No. 9, 1984, pp. 1304-1309.

${ }^{6}$ Kim, K.O., and Anderson, W. J., "Dynamic Condensation in Structural Dynamics Redesign," AIAA Journal, Vol. 22, No. 11, 1984, pp. 1616, 1617.

${ }^{7}$ Bernitsas, M. M., Hoff, C. J., and Kokarakis, J. E., "Nonlinear Inverse Perturbation in Structural Dynamics Redesign of Risers," Journal of Energy Resources Technology, ASME, Transactions, Vol. 107, No. 2, 1985, pp. 256-263.

${ }^{8}$ Hoff, C. J., and Bernitsas, M. M., "Dynamic Redesign of Marine Structures," Journal of Ship Research, Vol. 29, No. 4, 1985, pp. 285-295.

${ }^{9} \mathrm{Kim}$, J. H., and Bernitsas, M. M., "Redesign of Marine Structures by Perturbation," Journal of Marine Structures, Vol. 1, No. 2, 1988, pp. 139 183.

${ }^{10}$ Bernitsas, M. M., and Kang, B. S., "Admissible Large Perturbations in Structural Redesign," AIAA Journal, Vol. 29, No. 1, 1991, pp. 104-113.

${ }^{11}$ Bernitsas, M. M., and Tawekal, R. L., "Structural Model Correlation Using Large Admissible Perturbations in Cognate Space," AIAA Journal, Vol. 29, No. 12, 1991, pp. 2222-2232.

${ }^{12}$ Tawekal, R. L., and Bernitsas, M. M., "Finite Element Model Correlation for Offshore Structures," Proceedings of the 10th International OMAE
Conference, Norway, June 1991.

${ }^{13}$ Ting, T., Ojalvo, I. U., and Chen, T. L. C., "A Robust Modal Correlation Procedure for Large-Scale Structures," The 7th International Modal Analysis Conference, Vol. 1, Las Vegas, Nevada, 1989.

${ }^{14}$ Twomey, W. J., Chen, T. L. C., Ojalvo, I. U., and Ting, T., "A General Method for Modifying a Finite Element Model to Correlate with Modal Test Data," Journal of the American Helicopter Society, July 1991, pp. 4858 .

${ }^{15}$ Kang, B. S., Beyko, E., and Bernitsas, M. M., "Invariant and Consistent Redundancy by Large Admissible Perturbations," Journal of Marine Structures, Vol. 5, No. 1, 1992, pp. 23-70.

${ }^{16}$ Bernitsas, M. M., Kang, B. S., and Tawekal, R., "RESTRUCT Version 3.0: A Program for REdesign of STRUCTures," Publication No. 312, Dept. of Naval Architecture and Marine Engineering, Univ. of Michigan, Ann Arbor, MI, Oct. 1989.

${ }^{17}$ Bernitsas, M. M., Beyko, E., Rim, C. W., and Alzahabi, B., "Finite Element Structural Redesign by Large Admissible Perturbations," Applied Ocean Research, Vol. 14, No. 4, 1992, pp. 219-230.

${ }^{18}$ Beyko, E., and Bernitsas, M. M., "Reliability of Large Scale Structures by Large Admissible Perturbations," Journal of Offshore Mechanics and Arctic Engineering, ASME Transactions, Vol. 115, No. 3, 1993, pp. 167-178.

${ }^{19}$ Alzahabi, B., and Bernitsas, M. M., "Cylindrical Shell Redesign by Large Admissible Perturbations," Proceedings of the ASCE 9th Engineering Mechanics Conference, Texas A\&M Univ. (College Station, TX), May 1992, pp. 200-203.

${ }^{20}$ Gans, H. D., and Anderson, W. J., "Structural Optimization Incorporating Centrifugal and Coriolis Effects," Proceedings of the AIAA/ASME/ ASCE/AHS/ASC 30th Structures, Structural Dynamics, and Materials Conference, AIAA, Washington, DC, 1989, pp. 1311-1320.

${ }^{21}$ Haug, E. J., Choi, K. K., and Komkov, V., Design Sensitivity Analysis of Structural Systems, Academic Press, New York, 1986.

${ }^{22}$ Brockman, R. A., and Lung, F. Y., "Sensitivity Analysis with Plate and Shell Finite Elements," International Journal for Numerical Methods in Engineering, Vol. 26, No. 5, 1988, pp. 1129-1143.

${ }^{23}$ Vanderplaats, G. N., Miura, M., Nagendra, G., and Wallerstein, D., "Structural Synthesis Using MSC/NASTRAN," Computers in Engineering, Proceedings of the International Computers in Engineering Conference and Exhibit, 1989, pp. 211-218.

${ }^{24}$ Haftka, R. T., Gürdal, Z., and Kamat, M. P., Elements of Structural Optimization, 2nd edition, Kluwer Academic Publishers, 1990.

${ }^{25}$ Erbatur, F., and Mengi, Y., "Optimal Design of Plates under the Influence of Dead Weight and Surface Loading," Journal of Structures and Machines, Vol. 5, No. 4, 1977, pp. 345-356.

${ }^{26}$ Prasad, B., and Haftka, R. T., "Optimal Structural Design with Plate Finite Elements," Journal of Structural Division, Vol. 105, ST11, 1979, pp. 2367-2382.

${ }^{27}$ Haftka, R. T., and Prasad, B., "Optimum Structural Design with Plate Bending Elements-A Survey," AIAA Journal, Vol. 19, No. 4, 1981, pp. 517-522.

${ }^{28}$ Optimization of Shells and Plates," Structural Optimization, Vol. 2: Mathematical Programming, edited by M. Save and W. Prager, Plenum Press, New York, 1985.

${ }^{29}$ Koski, J., Silvennoinen, R., and Lawo, M., "Multicriterion Plate Optimization," Structural Optimization, edited by G. I. N. Rosvany and B. L. Karihaloo, Proceedings of the IUTAM Symposium on Structural Optimization (Melbourne, Australia), Feb. 1988, pp. 159-167.

${ }^{30}$ Natake, H. G., "Minimum Modifications due to Dynamic Requirements; Comparison and Application," Computer Aided Optimum Design of Structures: Recent Advances, Proceedings of the First International Conference (Southampton, England, UK), edited by C. A. Brebbia and S. Hernadez, Computational Mechanics, Southampton, England, UK, 1989, pp. $31-40$.

${ }^{31}$ Lin, C.-C., and Liu, I.-W., "Optimal Design for Plate Structures with Buckling Constraints," AIAA Journal, Vol. 28, No. 5, 1990, pp. 951-953.

${ }^{32}$ Gill, P. E., Murray, W., Saunders, M. A., and Wright, M. H., "User's Guide for SOL/NPSOL: A Fortran Package for Nonlinear Programming," Dept. of Operations Research, Stanford Univ., Stanford, CA, 1983.

${ }^{33}$ Timoshenko, S., and Woinowsky-Krieger, S., Theory of Plates and Shells, McGraw-Hill, New York, 1959.

${ }^{34}$ Blevins, R. D., Formulas for Natural Frequency and Mode Shape, Krieger, Molabar, FL, 1984.

${ }^{35}$ Leissa, A.W., "The Free Vibration of Rectangular Plates," Journal of Sound and Vibration, Vol. 31, No. 3, 1973, pp. 257-293. 